\title{
DISJUNCTIVE MULTIPLE-CONCLUSION CONSEQUENCE RELATIONS
}

\begin{abstract}
The concept of multiple-conclusion consequence relation from [8] and [7] is considered. The closure operation $C$ assigning to any binary relation $r$ (defined on the power set of a set of all formulas of a given language) the least multipleconclusion consequence relation containing $r$, is defined on the grounds of a natural Galois connection. It is shown that the very closure $C$ is an isomorphism from the power set algebra of a simple binary relation to the Boolean algebra of all multiple-conclusion consequence relations.
\end{abstract}

Keywords: multiple-conclusion consequence relation, closure operation, Galois connection.

\section{Preliminaries}

Given a set $A$, any mapping $C: \wp(A) \longrightarrow \wp(A)$ such that for each $X, Y \subseteq$ $A, X \subseteq C(X), C(C(X)) \subseteq C(X)$ and $C$ is monotone: $X \subseteq Y \Rightarrow C(X) \subseteq$ $C(Y)$, is called a closure operation defined on the power set $\wp(A)$ of $A$. Any subset $\mathcal{B} \subseteq \wp(A)$ is said to be a closure system over $A$ (or of the complete lattice $(\wp(A), \subseteq))$, if for each $\mathcal{X} \subseteq \mathcal{B}, \cap \mathcal{X} \in \mathcal{B}$. Given a closure operation $C$ on $\wp(A)$, the set of all its fixed points called closed elements: $C l(C)=\{X \subseteq A: X=C(X)\}$, is a closure system over $A$. Conversely, given a closure system $\mathcal{B}$ over $A$, the mapping $C: \wp(A) \longrightarrow \wp(A)$ defined by $C(X)=\bigcap\{Y \in \mathcal{B}: X \subseteq Y\}$, is a closure operation on $\wp(A)$. The closure system $\mathcal{B}$ is just the set of all its closed elements. On the other hand, the closure system $C l(C)$ of all closed elements of a given closure 
operation $C$ defines, in that way, just the operation $C$. Thus, there is a one to one correspondence between the class of all closure operations defined on $\wp(A)$ and of all closure systems of $(\wp(A), \subseteq)$, in fact, it is a dual isomorphism between the respective complete lattices of all closure operations and closure systems (the poset $(\mathcal{C}(A), \leq$ ) of all closure operations defined on $\wp(A)$, where $C_{1} \leq C_{2}$ iff $C_{1}(X) \subseteq C_{2}(X)$ for each $X \subseteq A$, forms a complete lattice such that for any class $\mathcal{E} \subseteq \mathcal{C}(A)$ its infimum, inf $\mathcal{E}$, is a closure operation defined on $\wp(A)$ by $(\inf \mathcal{E})(X)=\bigcap\{C(X): C \in \mathcal{E}\})$. Any closure system $\mathcal{B}$ of $(\wp(A), \subseteq)$ forms a complete lattice with respect to the order $\subseteq$ such that $\inf \mathcal{X}=\bigcap \mathcal{X}$ and $\sup \mathcal{X}=C(\bigcup \mathcal{X})$, for each $\mathcal{X} \subseteq \mathcal{B}$, where $C$ is the closure operation corresponding to closure system $\mathcal{B}$. Given a family $\mathcal{X} \subseteq \wp(A)$, there exists the least closure system $\mathcal{B}$ of $(\wp(A), \subseteq)$ such that $\mathcal{X} \subseteq \mathcal{B}$. It is called a closure system generated by $\mathcal{X}$ and shall be denoted by $[\mathcal{X}]$. It is simply the intersection of all closure systems of $(\wp(A), \subseteq)$ containing $\mathcal{X}$ and is expressed by $[\mathcal{X}]=\{\cap \mathcal{Y}: \mathcal{Y} \subseteq \mathcal{X}\}$. The closure operation $C$ corresponding to closure system $[\mathcal{X}]$ is defined by $C(X)=\bigcap\{Y \in \mathcal{X}: X \subseteq Y\}$, any $X \subseteq A$.

When $A$ is a set of all formulae of a given formal language, a closure operation $C$ defined on $\wp(A)$ is called a consequence operation (in the sense of Tarski).

We shall apply here the standard (called sometimes archetypal) antimonotone Galois connection $(f, g)$ defined on the complete lattices $(\wp(A), \subseteq),(\wp(B), \subseteq)$ of all subsets of given sets $A, B$ by a binary relation $R \subseteq A \times B$ (cf. [3], a general theory is to be found for example in $[1,2,4])$. That is, $f: \wp(A) \longrightarrow \wp(B)$ and $g: \wp(B) \longrightarrow \wp(A)$ are the mappings defined for any $X \subseteq A, a \in A, Y \subseteq B, b \in B$ by

$b \in f(X)$ iff for all $x \in X,(x, b) \in R$,

$a \in g(Y)$ iff for all $y \in Y,(a, y) \in R$.

The following three facts are useful for our goals.

The compositions $f \circ g, g \circ f$ are closure operations on $\wp(A), \wp(B)$, respectively.

The set $C l(f \circ g)$ of all closed sets with respect to closure operation $f \circ g$ is the counterdomain of map $g:\{X \subseteq A: g(f(X))=X\}=\{g(Y): Y \subseteq B\}$ and similarly, $C l(g \circ f)=\{Y \subseteq B: f(g(Y))=Y\}=\{f(X): X \subseteq A\}$. 
The mapping $f$ restricted to $C l(f \circ g)$ is a dual isomorphism of the complete lattices $(C l(f \circ g), \subseteq),(C l(g \circ f), \subseteq)$ as well as the map g restricted to $\mathrm{Cl}(\mathrm{g} \circ \mathrm{f})$ is the inverse dual isomorphism.

\section{The concept of disjunctive multiple-conclusion con- sequence relation}

This what will be called here a disjunctive consequence relation recalls the concept of multiple-conclusion entailment or multiple-conclusion consequence relation $[7,8]$. In $[8$, p. 28] the following definition of multipleconclusion consequence relation was introduced. Let $V$ be a set of all formulae of a given language. For any $\mathcal{T} \subseteq \wp(V)$ a binary relation $\vdash_{\mathcal{T}}$ is defined on $\wp(V)$ by

(mc) $X \vdash_{\mathcal{T}} Y$ iff $\forall T \in \mathcal{T}(X \subseteq T \Rightarrow Y \cap T \neq \emptyset)$.

We say that $\vdash \subseteq \wp(V) \times \wp(V)$ is a multiple-conclusion consequence relation iff $\vdash=\vdash_{\mathcal{T}}$ for some $\mathcal{T} \subseteq \wp(V)$. Next the authors of [8] prove the theorem (2.1, p. 30):

A relation $\vdash$ is a multiple-conclusion consequence relation iff it satisfies the following conditions for any $X, Y \subseteq V$ :

(overlap) $X \cap Y \neq \emptyset \Rightarrow X \vdash Y$,

(dilution) $X \vdash Y, X \subseteq X^{\prime}, Y \subseteq Y^{\prime} \Rightarrow X^{\prime} \vdash Y^{\prime}$,

(cutforsets) $\forall S \subseteq V((\forall Z \subseteq S, X \cup Z \vdash Y \cup(S-Z)) \Rightarrow X \vdash Y)$.

Given $S \subseteq V$, the part $(\forall Z \subseteq S, X \cup Z \vdash Y \cup(S-Z)) \Rightarrow X \vdash Y$ of the condition (cutforsets) is called (cutfor $S$ ). In turn, (cutforformulae) denotes the family of all the conditions (cutfor $\{\alpha\}), \alpha \in V$ :

$$
\text { (cutfor }\{\alpha\}) X \vdash Y \cup\{\alpha\} \& X \cup\{\alpha\} \vdash Y \Rightarrow X \vdash Y,
$$

that is, stands to the cut rule of [5] from 1934. In general, granted (dilution), the conditions (cutforsets) and (cutforV) are equivalent (Theorem 2.2 in [8], p. 31). Moreover, when a binary relation $\vdash \subseteq \wp(V) \times \wp(V)$ satisfies not only (dilution) but also is compact, i.e fulfils the condition

(compactness) $X \vdash Y \Rightarrow$ there exist finite subsets $X^{\prime} \subseteq X, Y^{\prime} \subseteq Y$ such that $X^{\prime} \vdash Y^{\prime}$, 
both conditions (cutforsets), (cutforformulae) are equivalent (Theorem 2.9 in [8], p. 37).

The conditions (overlap),(dilution), (cutforformulae), under different names, were used to define on finite sets of formulas, the relation of multiple-conclusion entailment by D. Scott [7].

In [11] it was proved that when a family $\mathcal{T} \subseteq \wp(V)$ is a closure system over $V$, the consequence relation $\vdash_{\mathcal{T}}$ defined by $(m c)$, may be expressed by

(dis) $X \vdash_{\mathcal{T}} Y$ iff $Y \cap C_{\mathcal{T}}(X) \neq \emptyset$,

where $C_{\mathcal{T}}$ is the closure operation determined by closure system $\mathcal{T}$. As it is seen, given a set of premises $X$ some of conclusions of the consequence relation $\vdash_{\mathcal{T}}$ are conclusions of ordinary consequence operation $C_{\mathcal{T}}$ associated with the relation. So, one may say that the relation $\vdash_{\mathcal{T}}$ has a disjunctive character. It is worth to notice that in general, for arbitrary family $\mathcal{T} \subseteq \wp(V)$ only the implication $(\Leftarrow)$ from right to left holds true, where in case, $C_{\mathcal{T}}$ is the closure operation (consequence operation) determined by the family $\mathcal{T}$ (that is, by $[\mathcal{T}]$ - the least closure system over $V$ containing $\mathcal{T})$ : for a formula $\alpha \in V, \alpha \in C_{\mathcal{T}}(X)$ iff for any $T \in \mathcal{T}, X \subseteq T \Rightarrow \alpha \in T$.

Hereafter the consequence relations $\vdash_{\mathcal{T}}, \mathcal{T} \subseteq \wp(V)$ will be called disjunctive. Let $D R=\left\{\vdash_{\mathcal{T}}: \mathcal{T} \subseteq \wp(V)\right\}$.

\section{Galois connection for disjunctive consequence rela- tion}

Taking into account the very definition of disjunctive consequence relation from the previous section (cf. $(m c))$, the following Galois connection $(f, g)$ should be considered. Put $R \subseteq \wp(V)^{2} \times \wp(V)$ of the form $((X, Y), T) \in R$ iff $X \subseteq T \Rightarrow Y \cap T \neq \emptyset$. So $f:(\wp(\wp(V) \times \wp(V)), \subseteq) \longrightarrow(\wp(\wp(V)), \subseteq)$, $g:(\wp(\wp(V)), \subseteq) \longrightarrow(\wp(\wp(V) \times \wp(V)), \subseteq)$ are defined for any relation $r \subseteq \wp(V) \times \wp(V)$ and any family $\mathcal{T} \subseteq \wp(V)$ by

$T \in f(r)$ iff for all $X, Y \subseteq V$ such that $(X, Y) \in r, X \subseteq T$ implies that $Y \cap T \neq \emptyset$, any $T \subseteq V$,

$(X, Y) \in g(\mathcal{T})$ iff for all $T \in \mathcal{T}, X \subseteq T$ implies that $Y \cap T \neq \emptyset$, any $X, Y \subseteq V$. 
In more handy formulation,

(1) $T \in f(r)$ iff $\forall X, Y \subseteq V(X \subseteq T \subseteq-Y \Rightarrow(X, Y) \notin r)$,

(2) $(X, Y) \in g(\mathcal{T})$ iff $\forall T \subseteq V(X \subseteq T \subseteq-Y \Rightarrow T \notin \mathcal{T})$,

where "-" is the operation of complementation in the Boolean algebra of all subsets of $V$.

Let us put $C=f \circ g$ and $C^{\prime}=g \circ f$, that is, $C$ is a closure operation defined on $\wp(\wp(V) \times \wp(V))$ assigning to each binary relation $r$ defined on $\wp(V)$ the least relation from $D R$ containing $r$ (the operation $C$ is the counterpart of closure introduced in [6, p. 1006, definition 3.1] for Scott's multiple-conclusion relations from [7]); in turn $C^{\prime}$ is a closure operation whose closed sets correspond via dual isomorphism $f$ restricted to $D R$ to disjunctive consequence relations. Using (1) and (2) we obtain that for any binary relation $r \subseteq \wp(V) \times \wp(V),(X, Y) \in C(r)$ iff $(X, Y) \in g(f(r))$ iff $\forall T \subseteq V(X \subseteq T \subseteq-Y \Rightarrow T \notin f(r))$ iff $\forall T \subseteq V(X \subseteq T \subseteq-Y \Rightarrow$ $\exists U, Z \subseteq V(U \subseteq T \subseteq-Z \&(U, Z) \in r))$. Finally,

(3) $(X, Y) \in C(r)$ iff $[X,-Y] \subseteq \bigcup\{[U,-Z]:(U, Z) \in r\}$,

where for any $X, Y \subseteq V,[X, Y]=\{U \subseteq V: X \subseteq U \subseteq Y\}$. However, the equivalence:

(4) $(X, Y) \in C(r)$ iff $\forall T \subseteq V(X \subseteq T \subseteq-Y \Rightarrow T \notin f(r))$,

is also interesting since from it one may derive that for any set $T \subseteq V$ and any binary relation $r \subseteq \wp(V) \times \wp(V)$,

(5) $T \in f(r)$ iff $(T,-T) \notin C(r)$.

Similarly, for any family $\mathcal{T} \subseteq \wp(V): T \in C^{\prime}(\mathcal{T})$ iff $T \in f(g(\mathcal{T}))$ iff $\forall X, Y \subseteq V\left(X \subseteq T \subseteq-Y \Rightarrow \exists T^{\prime} \subseteq V\left(X \subseteq T^{\prime} \subseteq-Y \& T^{\prime} \in \mathcal{T}\right)\right)$ iff $T \in \mathcal{T}$. In this way, $C^{\prime}$ is the identity mapping on $\wp(\wp(V))$ so $C l\left(C^{\prime}\right)=$ $C l(g \circ f)=\wp(\wp(V))$. On the other hand, $C l(C)=C l(f \circ g)=\{g(\mathcal{T}): \mathcal{T} \subseteq$ $\wp(V)\}=\left\{\vdash_{\mathcal{T}}: \mathcal{T} \subseteq \wp(V)\right\}=D R$. Thus we have the following corollary.

COROLlarY. The mapping $f$ restricted to $D R$ (that is $f$ defined for each $r \in D R$ by $f(r)=\{T \subseteq V:(T,-T) \notin r\}$ due to (5)) is a dual isomorphism of the complete lattices $(D R, \subseteq),(\wp(\wp(V)), \subseteq)$ and the mapping $g$ is the inverse dual isomorphism. 
This result, obtained first in [11] without application of Galois connection, can be strengthened (cf. also [11]) to a dual isomorphism of complete and atomic Boolean algebras $\left(D R, \cap, \vee,-, \vdash_{0}, \wp(V)^{2}\right),(\wp(\wp(V)), \cap, \cup,-, \emptyset$, $\wp(V)$ ), by equipping the family $D R$ of disjunctive relations with the operation of Boolean complementation - in such a way that the dual isomorphism of complete lattices preserves it : $-r=-g(f(r))=g(\wp(V)-f(r))=$ $g(\{T \subseteq V:(T,-T) \in r\})$. Here for any $r_{1}, r_{2} \in D R, r_{1} \vee r_{2}=C\left(r_{1} \cup r_{2}\right)$ and $\vdash_{0}=g(\wp(V))=\{(X, Y): X \cap Y \neq \emptyset\}$ is the least disjunctive relation.

\section{Isomorphism theorem for disjunctive consequence relations}

Let us put $\mathcal{R}_{0}=\{(T,-T): T \subseteq V\}$. Consider the mapping $p: \wp\left(\mathcal{R}_{0}\right) \longrightarrow$ $\wp(\wp(V))$ defined by $p(\rho)=\{T \subseteq V:(T,-T) \in \rho\}$. It is obvious that $p$ is a Boolean and complete isomorphism of Boolean algebras $\left(\wp\left(\mathcal{R}_{0}\right), \cap, \cup,-, \emptyset\right.$, $\left.\mathcal{R}_{0}\right), \quad(\wp(\wp(V)), \cap, \cup,-, \emptyset, \wp(V))$. Consider the following composition of mappings:

$$
\wp\left(\mathcal{R}_{0}\right) \ni \rho \longmapsto p(\rho) \longmapsto \wp(V)-p(\rho) \longmapsto g(\wp(V)-p(\rho)) \in D R .
$$

The correspondence $\wp(\wp(V)) \ni \mathcal{T} \longmapsto \wp(V)-\mathcal{T}$ is obviously a dual Boolean complete isomorphism from $(\wp(\wp(V)), \cap, \cup,-, \emptyset, \wp(V))$ onto itself. So the composition $\wp\left(\mathcal{R}_{0}\right) \ni \rho \longmapsto g(\wp(V)-p(\rho)) \in D R$ (one isomorphism and two dual isomorphisms are here composed) is a complete Boolean isomorphism from $\left(\wp\left(\mathcal{R}_{0}\right), \cap, \cup,-, \emptyset, \mathcal{R}_{0}\right)$ onto $\left(D R, \cap, \vee,-, \vdash_{0}, \wp(V)^{2}\right)$.

Using (2) one may calculate the value of that isomorphism on a $\rho \subseteq \mathcal{R}_{0}$ : for any $X, Y \subseteq V,(X, Y) \in g(\wp(V)-p(\rho))$ iff $[X,-Y] \subseteq p(\rho)$. Moreover, from (3) we have

(6) $(X, Y) \in C(\rho) \quad$ iff $\quad[X,-Y] \subseteq \bigcup\{[T, T]:(T,-T) \in \rho\} \quad$ iff $[X,-Y] \subseteq p(\rho)$.

Therefore, for any $\rho \subseteq \mathcal{R}_{0}, C(\rho)=g(\wp(V)-p(\rho))$. Furthermore, one may consider the inverse isomorphism as the following composition:

(5)) $\longmapsto r \cap \mathcal{R}_{0}$.

$D R \ni r \longmapsto f(r) \longmapsto \wp(V)-f(r)=\{T \subseteq V:(T,-T) \in r\} \quad$ (by

In this way the following result is proved. 
Proposition. The closure operation $C$ (assigning to each binary relation $r$ defined on $\wp(V)$ the least disjunctive relation containing $r$ ) restricted to the power set of $\mathcal{R}_{0}=\{(T,-T): T \subseteq V\}$ is a Boolean and complete isomorphism from the power set algebra $\left(\wp\left(\mathcal{R}_{0}\right), \cap, \cup,-, \emptyset, \mathcal{R}_{0}\right)$ onto atomic and complete Boolean algebra $\left(D R, \cap, \vee,-, \vdash_{0}, \wp(V)^{2}\right)$ of all disjunctive relations defined on the language $V$. The inverse isomorphism, say $h: D R \longrightarrow \wp\left(\mathcal{R}_{0}\right)$ is defined by $h(r)=r \cap \mathcal{R}_{0}$. In this way, for any $r \in D R$ and $\rho \subseteq \mathcal{R}_{0}, r=C\left(r \cap \mathcal{R}_{0}\right)$ and $\rho=C(\rho) \cap \mathcal{R}_{0}$.

\section{Some applications}

Applying (6) one may show that for any $T_{1}, T_{2} \subseteq V$ such that $T_{1} \subseteq T_{2}$ and for any $X, Y \subseteq V$,

(7) $(X, Y) \in C\left(\left\{(T,-T): T \in\left[T_{1}, T_{2}\right]\right\}\right)$ iff either $X \vdash_{0} Y$ or $T_{1} \subseteq X \subseteq-Y \subseteq T_{2}$.

In particular, using (7) and Proposition, one may find a form of atoms in the Boolean algebra $\left(D R, \cap, \vee,-, \vdash_{0}, \wp(V)^{2}\right)$ of all disjunctive relations. Let us take any atom $\{(T,-T)\}, T \subseteq V$, of $\left(\wp\left(\mathcal{R}_{0}\right), \cap, \cup,-, \emptyset, \mathcal{R}_{0}\right)$. Then the corresponding atom in the Boolean algebra of all disjunctive relations is of the form:

(8) $C(\{(T,-T)\})=\vdash_{0} \cup\{(T,-T)\}$.

The coatoms of $\left(D R, \cap, \vee,-, \vdash_{0}, \wp(V)^{2}\right)$ are much more interesting. Take any $T \subseteq V$. Then the corresponding coatom in this Boolean algebra to the coatom $\mathcal{R}_{0}-\{(T,-T)\}$ of $\left(\wp\left(\mathcal{R}_{0}\right), \cap, \cup,-, \emptyset, \mathcal{R}_{0}\right)$ is, due to $(6)$ and $(m c)$, of the form

(9) $(X, Y) \in C\left(\mathcal{R}_{0}-\{(T,-T)\}\right)$ iff $[X,-Y] \subseteq \wp(V)-\{T\}$ iff either $X \nsubseteq T$ or $Y \cap T \neq \emptyset$ iff $X \vdash_{\{T\}} Y$.

More figuratively,

(10) $C\left(\mathcal{R}_{0}-\{(T,-T)\}\right)=\vdash_{\{T\}}=\bigcup\{[(\{\alpha\}, \emptyset)): \alpha \notin T\} \cup \bigcup\{[(\emptyset,\{\alpha\})):$ $\alpha \in T\}$,

where for any $X, Y \subseteq V,[(X, Y))=\left\{\left(X^{\prime}, Y^{\prime}\right) \in \wp(V)^{2}: X \subseteq X^{\prime} \&\right.$ $\left.Y \subseteq Y^{\prime}\right\}$.

The following lemma provides a useful characteristics of coatoms. 
Lemma. For any $\vdash \in D R$ and $T \subseteq V, \vdash=\vdash_{\{T\}}$ iff for each $\alpha \in V,(\emptyset \vdash$ $\{\alpha\} \quad$ iff $\alpha \in T)$ and $(\{\alpha\} \vdash \emptyset$ iff $\alpha \notin T)$.

Proof. Consider any disjunctive relation $\vdash$ and $T \subseteq V$.

$(\Rightarrow)$ : By $(10)$.

$(\Leftarrow)$ : Assume that for each $\alpha \in V,(\emptyset \vdash\{\alpha\}$ iff $\alpha \in T)$ and $(\{\alpha\} \vdash \emptyset$ iff $\alpha \notin T)$. First we show that $\vdash_{\{T\}} \subseteq \vdash$. So suppose that $X \vdash_{\{T\}} Y$, that is, either $X \nsubseteq T$ or $Y \cap T \neq \emptyset$. In the first case, from the assumption it follows that $\{\alpha\} \vdash \emptyset$ for some $\alpha \in X$ so $X \vdash Y$ by (dilution). In the second case, analogously, $\emptyset \vdash\{\alpha\}$ for some $\alpha \in Y$ so $X \vdash Y$. Now notice that $\vdash_{\{T\}}$ is a coatom in the Boolean algebra of all disjunctive relations, therefore the inclusion $\vdash_{\{T\}} \subseteq \vdash$ implies that $\vdash_{\{T\}}=\vdash$ or $\vdash=\wp(V)^{2}$. Since the relation $\wp(V)^{2}$ does not satisfy the assumption we obtain $\vdash_{\{T\}}=\vdash$.

The coatoms in the Boolean algebra of all disjunctive consequence relations are easily expressible in terms of [7]. In order to show this let us apply the definition from [7, p. 416], for any disjunctive relation. A relation $\vdash \in D R$ is said to be consistent (complete) iff for any $\alpha \in V$, either $\emptyset \nvdash\{\alpha\}$ or $\{\alpha\} \forall \emptyset$ (for any $\alpha \in V$, either $\emptyset \vdash\{\alpha\}$ or $\{\alpha\} \vdash \emptyset$ ). In this way, for any $\vdash \in D R$,

(11) $\vdash$ is consistent and complete iff for any $\alpha \in V, \emptyset \vdash\{\alpha\}$ iff $\{\alpha\} \forall \emptyset$.

FACT. For any $\vdash \in D R, \vdash$ is consistent and complete iff for some $T \subseteq$ $V, \vdash=\vdash_{\{T\}}$.

Proof. Consider any disjunctive relation $\vdash$.

$(\Rightarrow)$ : Assume that $\vdash$ is consistent and complete. Put $T=\{\alpha \in V: \emptyset \vdash$ $\{\alpha\}\}$. Then from the assumption and (11) it follows that $-T=\{\alpha \in V$ : $\{\alpha\} \vdash \emptyset\}$. In this way, $\vdash=\vdash_{\{T\}}$ due to Lemma.

$(\Leftarrow)$ : Immediately from Lemma and (11).

In the light of this fact, the result of [7] that any multiple-conclusion consequence relation is an intersection of all consistent and complete relations containing it, becomes absolutely clear. Since for every $\vdash \in D R$, the identity $\vdash=\bigcap\left\{\vdash_{\{T\}}: \vdash \subseteq \vdash_{\{T\}}\right\}$ holds. In turn, the latter connection is an obvious consequence of the following one: $\rho=\bigcap\left\{\mathcal{R}_{0}-\{(T,-T)\}\right.$ : $(T,-T) \notin \rho\}$, any $\rho \subseteq \mathcal{R}_{0}$ (implying together with Proposition and (9) that $C(\rho)=\bigcap\left\{C\left(\mathcal{R}_{0}-\{(T,-T)\}\right): \rho \subseteq \mathcal{R}_{0}-\{(T,-T)\}\right\}=\bigcap\left\{\vdash_{\{T\}}\right.$ : $\left.\left.C(\rho) \subseteq \vdash_{\{T\}}\right\}\right)$. 
Notice that the power set $\wp\left(\mathcal{R}_{0}\right)$ is closed on the operation $\sim$ of taking the converse relation. Applying (6) for a given $\rho \subseteq \mathcal{R}_{0}$ we have $(X, Y) \in$ $C\left(\rho^{\sim}\right)$ iff $[X,-Y] \subseteq p\left(\rho^{\sim}\right)$ iff $[X,-Y] \subseteq\{-T: T \in p(\rho)\}$ iff $[Y,-X] \subseteq$ $p(\rho)$ iff $(Y, X) \in C(\rho)$ iff $(X, Y) \in C(\rho)^{\sim}$. Hence, $C\left(\rho^{\sim}\right)=C(\rho)^{\sim}$ so the operation $\sim$ is preserved under the isomorphism $C$ and the set $D R$ is closed on this operation. Denoting for a given family $\mathcal{T} \subseteq \wp(V), \mathcal{T}^{\sim}=$ $\{-T: T \in \mathcal{T}\}$ we have $g\left(\mathcal{T}^{\sim}\right)=g(\mathcal{T})^{\sim}$ due to $(2)$, that is, in terms of $(m c)$ :

(12) $\vdash_{\mathcal{T} \sim}=\vdash_{\mathcal{\mathcal { T }}}$.

Given $\vdash \in D R$ the relation $\vdash^{\sim}$ could be called dual with respect to $\vdash$. For example, assume that $V$ is the set of all formulas of propositional language equipped with the standard connectives $\neg, \wedge, \vee, \rightarrow$ and let $V a l$ be the set of all Boolean valuations of the language into $\{0,1\}$. Consider the disjunctive relation $\vdash_{\mathcal{T}_{M a x}}$ determined (according to $(m c)$ ) by the family of all maximal theories of classical propositional logics $\mathcal{T}_{\text {Max }}=\left\{T_{v}: v \in \mathrm{Val}\right\}$, where for each $v \in$ Val, $T_{v}=\{\alpha \in V: v(\alpha)=1\}$ (cf. also [9, p. 242, definition 1]):

$X \vdash_{\mathcal{T}_{M a x}} Y$ iff $\forall v \in \operatorname{Val}\left(X \subseteq T_{v} \Rightarrow Y \cap T_{v} \neq \emptyset\right)$ iff $\forall v \in \operatorname{Val}(v[X] \subseteq$ $\{1\} \Rightarrow \exists \alpha \in Y, v(\alpha)=1)$.

The dual relation with respect to $\vdash_{\mathcal{T}_{M a x}}$ is, according to (12), determined by the family $\mathcal{T}_{\text {Max }}=\{\{\alpha \in V: v(\alpha)=0\}: v \in V a l\}$ (notice that the consequence operation corresponding to the closure system $\left[\mathcal{T}_{M} \tilde{M}_{a x}\right]$ over $V$ is dual in the sense of Wójcicki [10] with respect to the consequence operation of classical propositional logic, that is, corresponding to the closure system $\left.\left[\mathcal{T}_{\text {Max }}\right]\right)$. One may consider the dual disjunctive relation with respect to a coatom $\vdash_{\{T\}}, T \subseteq V$ which is the coatom $\vdash_{\{-T\}}$ (cf. also (10)). In particular $\vdash_{\left\{-T_{v}\right\}}, v \in V a l$ is considered in [9, p. 245, definition 3].

\section{References}

[1] T. S. Blyth, Lattices and Ordered Algebraic Structures, Springer, 2005.

[2] K. Denecke, M. Erné, S. L. Wismath (eds.), Galois Connections and Applications, Kluwer, 2004.

[3] F. Domenach, B. Leclerc, Biclosed binary relations and Galois connections, Order, Vol. 18 (2001), pp. 89-104. 
[4] M. Erné, J. Koslowski, A. Melton, G. E. Strecker, A Primer on Galois Connections, Annals of the New York Academy of Sciences, Vol. 704 (1993), pp. 103-125.

[5] G. K. E. Gentzen, Untersuchungen uber das logische Schliesen. I, Mathematische Zeitschrift, Vol. 39 (1934), pp. 176-210, [English translation: Investigation into Logical Deduction, [in:] M. E. Szabo, The collected Works of Gerhard Gentzen, North Holland, 1969, pp. 68-131.]

[6] G. Payette, P. K. Schotch, Remarks on the Scott-Lindenbaum Theorem, Studia Logica, Vol. 102 (2014), pp. 1003-1020.

[7] D. Scott, Completeness and axiomatizability in many-valued logic, Proceedings of Symposia in Pure Mathematics, Vol. 25 (Proceedings of the Tarski Symposium), American Mathematical Society 1974, pp. $411-435$.

[8] D. J. Shoesmith, T. J. Smiley, Multiple-conclusion Logic, Cambridge 1978.

[9] T. Skura, A. Wiśniewski, A system for proper multiple-conclusion entailment, Logic and Logical Philosophy, Vol. 24 (2015), pp. 241-253.

[10] R. Wójcicki, Dual counterparts of consequence operations, Bulletin of the Section of Logic, Vol. 2 (1973), pp. 54-56.

[11] J. Zygmunt, An Essay in Matrix Semantics for Consequence Relations, Wydawnictwo Uniwersytetu Wrocławskiego, 1984.

University of Łódź

Department of Logic

Lindleya 3/5, 90-131 Łódź

e-mail: marek.nowak@filozof .uni.lodz.pl 